\title{
On conformal field theories in four dimensions
}

\section{Citation}

Lawrence, Albion, Nikita Nekrasov, and Cumrun Vafa. 1998. "On Conformal Field Theories in Four Dimensions." Nuclear Physics B 533 (1-3): 199-209. https://doi.org/10.1016/ s0550-3213(98)00495-7.

\section{Permanent link}

http://nrs.harvard.edu/urn-3:HUL.InstRepos:41385079

\section{Terms of Use}

This article was downloaded from Harvard University's DASH repository, and is made available under the terms and conditions applicable to Other Posted Material, as set forth at http:// nrs.harvard.edu/urn-3:HUL.InstRepos:dash.current.terms-of-use\#LAA

\section{Share Your Story}

The Harvard community has made this article openly available.

Please share how this access benefits you. Submit a story.

\section{Accessibility}


hep-th/9803015

HUTP-98/A015

ITEP-TH-15/98

\title{
On Conformal Field Theories in Four Dimensions
}

\author{
Albion Lawrence ${ }^{2}$, Nikita Nekrasov ${ }^{1,2}$ and Cumrun Vafa $^{2}$ \\ ${ }^{1}$ Institute of Theoretical and Experimental Physics, 117259, Moscow, Russia \\ ${ }^{2}$ Lyman Laboratory of Physics, Harvard University, Cambridge, MA 02138
}

\begin{abstract}
Extending recent work of Kachru and Silverstein, we consider "orbifolds" of 4-dimensional $\mathcal{N}=4 S U(n)$ super-Yang-Mills theories with respect to discrete subgroups of the $S U(4)$ $R$-symmetry which act nontrivially on the gauge group. We show that for every discrete subgroup of $S U(4)$ there is a canonical choice of imbedding of the discrete group in the gauge group which leads to theories with a vanishing one-loop beta-function. We conjecture that these give rise to (generically non-supersymmetric) conformal theories. The gauge group is $\otimes_{i} S U\left(N n_{i}\right)$ where $n_{i}$ denote the dimension of the irreducible representations of the corresponding discrete group; there is also bifundamental matter, dictated by associated quiver diagrams. The interactions can also be read off from the quiver diagram. For $S U(3)$ and $S U(2)$ subgroups this leads to superconformal theories with $\mathcal{N}=1$ and $\mathcal{N}=2$ respectively. In the $\mathcal{N}=1$ case we prove the vanishing of the beta functions to two loops.
\end{abstract}

March 1998 


\section{Introduction}

It has been a longstanding problem in quantum field theories to obtain non-trivial four dimensional theories with vanishing beta functions which lead to conformal theories [1]. With recent progress in understanding of supersymmetric gauge theories we have learned how superconformal theories can arise in certain cases. However, not much progress has been made for the non-supersymmetric case, due in particular to an absence of nonrenormalization theorems. Very recently, motivated by connections between the gauge systems on branes and their supergravity realizations at large $N$ [2] [3], Kachru and Silverstein [四 (following the discussions in refs [5] [6] [7] [8]) considered an "orbifold" construction of gauge theories in four dimensions (with or without supersymmetry) and argued they should be related to conformal fixed points (at least in large $N$ ) . The aim of this paper is to extend their construction. We find a large set of proposed gauge theories in four dimensions, which we conjecture will lead to conformal fixed points. As a first check we show that they all have vanishing one-loop beta functions for the gauge couplings.

We construct for every discrete subgroup $\Gamma \subset S U(4)$ a gauge theory consisting of gauge group $G=\otimes S U\left(N n_{i}\right)$ where $N$ is an arbitrary integer and $n_{i}$ denote the dimensions of the irreducible representations of $\Gamma$. The matter and interactions can be read off from an associated "quiver" diagram. It consists of one node for each irreducible representation of $\Gamma$ and fermionic and bosonic arrows connecting the nodes according to how the 4 and 6 dimensional represenations of $\Gamma$ (inherited from $S U(4)$ ) act on each irreducible representation. To each bosonic/fermionic arrow from node $i$ to node $j$ we associate a bifundamental $\left(N n_{i}, \overline{N n_{j}}\right)$ scalar/Weyl fermion (for $i=j$ this corresponds to an adjoint representation). There is a Yukawa coupling for each triangle on the quiver, consisting of two fermionic arrows and a bosonic arrow; and quartic scalar interactions for each square on the quiver, consisting of four bosonic arrows. The resulting gauge theory is chiral if and only if $\Gamma$ is a complex subgroup of $S U(4)$, i.e. if $\mathbf{4}$ and $\overline{\mathbf{4}}$ of $S U(4)$ are inequivalent representations of $\Gamma$. If $\Gamma \subset S U(3)$ the resulting theory is an $\mathcal{N}=1$ theory and if $\Gamma \subset S U(2)$ it is an $\mathcal{N}=2$ theory. In such cases we can use the supersymmery structure to discuss a reduced quiver (with only one kind of arrow, consisting of multiplication with $\mathbf{3}$ of $S U(3)$ and 2 of $S U(2))$.

1 The $\mathcal{N}=1$ example considered in [由] had previously been considered in connection with finding a finite $\mathcal{N}=1$ theory in [9]. 
If $\Gamma \subset S U(2)$ we obtain an $\mathcal{N}=2$ theory. In fact it is known that this gives us all possible superconformal theories with gauge group $\otimes_{i} S U\left(N n_{i}\right)$ and bifundamental matter [10], i.e. those theories which arise from quiver diagrams corresponding to affine ADE Dynkin diagrams. Similarly, we conjecture that for the $\mathcal{N}=1,0$ cases the theories we construct, we also exhaust the possibilities for superconformal theories with gauge group $\otimes_{i} S U\left(N n_{i}\right)$ and bifundamental matter. In all cases, we can provide some geometric intuition for the meaning of the choices we have made for "orbifolding" based on the geometry of branes imbedded in a string background.

\section{Projections preserving conformal invariance}

Kachru and Silverstein suggested that if one studies $N$ D3-branes on various orbifolds of $\mathbb{R}^{6}$ and follows the conjecture of [3] (i.e. for values of $N, g_{s}$, and $M_{s}$ for which that picture makes sense), then one finds that one is studying an orbifold of $A d S_{5} \times S^{5}$ where the orbifold group acts only on the $S^{5}$ factor. Since the conformal group of the field theory is identified with the isometry group of $A d S_{5}$, these orbifolds should lead to conformal field theories on the D3-branes.

\subsection{Projection of the field theory: general story}

For the purposes of understanding the field content, one can simply work with the perturbative open string theory on the D-branes, as was done in [5] [6] for the $\mathcal{N}=2$ case and in 8 for the $\mathcal{N}=1$ case. Since we would like to keep our discussion as close to field theory as possible, we will abstract the discussion in [5] below and discuss general projections on the fields of the original $\mathcal{N}=4$ theory. Within this abstract setup, we can ask which projections lead to vanishing one-loop beta-functions for the gauge coupling. Of course, in general the beta functions for other couplings must be checked as well.

We will start with an $U(n) \mathcal{N}=4$ field theory. As is well known (see for example [11] for a discussion with references), this theory has an $\operatorname{Spin}(6)=S U(4) R$-symmetry group (the transverse rotation group of the D3-brane in the perturbative open string picture, or the rotation group of the $S^{5}$ in [3].) It contains the gauge bosons $A_{I J}(I, J=1, \ldots, n)$ which are singlets of $S O(6)$; adjoint Weyl fermions $\Psi_{I J}^{\alpha}$ with $\alpha$ in a 4 of $S U(4)$; and adjoint scalars $\Phi_{I J}^{m}$ with $m$ in the $\mathbf{6}$ of $S O(6)$. We now wish to pick a discrete subgroup $\Gamma$ of $\operatorname{Spin}(6)$ which acts nontrivially on the gauge group; such an action corresponds to the action of the orbifold group on the Chan-Paton factors of the open strings [12][5]. 
Let $\left\{\mathbf{r}_{i}\right\}$ be the set of unitary irreducible representations of $\Gamma$. We can specify the action by breaking up the indices $I$ into various representations $\mathcal{R}_{i}=\mathbb{C}^{N_{i}} \mathbf{r}_{i}$ of $\Gamma$ such that $\sum_{i} N_{i} \operatorname{dim}\left(\mathbf{r}_{i}\right)=n$, where $N_{i}$ denotes the number of times the representation $\mathbf{r}_{i}$ appears in this decomposition. Here $\Gamma$ acts trivially on $\mathbb{C}^{N_{i}}$. Note that for projections which break only part of the supersymmetry, $\Gamma$ will lie in $S U(2)$, preserving $\mathcal{N}=2$ or $S U(3)$, preserving $\mathcal{N}=1$.

We now consider a modified theory whose fields correspond to keeping $\Gamma$-invariant fields of the original theory, together with the terms in the action containing them. This is not orbifolding in the usual sense of that word as it is not gauging a discrete symmetry. However, we sometimes will continue to refer to this as orbifolding or projecting the original theory; the justification of this terminology comes from the fact that in string context such theories arise upon orbifolding string backgrounds. One may write $U(n)$ adjoint fields which are singlets under the broken R-symmetry as homomorphisms from $\mathbb{C}^{n}$ to itself, or as $\mathbb{C}^{n} \otimes\left(\mathbb{C}^{n}\right)^{*}$. The effect of this projection is easily derived:

$$
\begin{aligned}
& \operatorname{Hom}\left(\mathbb{C}^{n}, \mathbb{C}^{n}\right)^{\Gamma}=\bigoplus_{i, j} \operatorname{Hom}\left(\mathcal{R}_{i}, \mathcal{R}_{j}\right)^{\Gamma}= \\
& \bigoplus_{i, j}\left(\mathbb{C}^{N_{i}} \otimes\left(\mathbb{C}^{N_{j}}\right)^{*} \otimes \mathbf{r}_{i} \otimes \mathbf{r}_{j}^{*}\right)^{\Gamma}=\bigoplus_{i} \mathbb{C}^{N_{i}} \otimes\left(\mathbb{C}^{N_{i}}\right)^{*},
\end{aligned}
$$

where the superscript $\Gamma$ means keeping only the trivial representations in the decomposition with respect to the irreps of $\Gamma$. Thus $R$-charge singlets break up into adjoints of $U\left(N_{i}\right)$; in particular the unbroken gauge group is

$$
G_{\text {proj }}=\otimes_{i} U\left(N_{i}\right)
$$

The $U(1)$ factors decouple at low energies, so we will in fact consider

$$
G_{\text {proj }}=\otimes_{i} S U\left(N_{i}\right)
$$

The projection for fields carrying $R$-charge is a bit more complicated. Let us examine fields transforming under the R-symmetry. For each (not necessarily irreducible) representation $\mathcal{R}$ of $\Gamma$ define the coefficients $a_{i j}^{\mathcal{R}}$ by the equations

$$
\mathcal{R} \otimes \mathbf{r}_{i}=\oplus_{j} a_{i j}^{\mathcal{R}} \mathbf{r}_{j}
$$


Then we may describe the projection of an adjoint field with $R$-charge as

$$
\begin{aligned}
& \left(\mathcal{R} \otimes \operatorname{Hom}\left(\mathbb{C}^{n}, \mathbb{C}^{n}\right)\right)^{\Gamma}=\bigoplus_{i, j}\left(\mathcal{R} \otimes \mathcal{R}_{i} \otimes \mathcal{R}_{j}^{*}\right)^{\Gamma}= \\
& \bigoplus_{i, j, k} a_{i j}^{\mathcal{R}}\left(\mathcal{R}_{j} \otimes \mathcal{R}_{k}^{*}\right)^{\Gamma}=\bigoplus_{i, j} a_{i, j}^{\mathcal{R}} \mathbb{C}^{N_{i}} \otimes\left(\mathbb{C}^{N_{i}}\right)^{*}
\end{aligned}
$$

Now let 4,6 denote representations of $\Gamma$ coming from the fundamental and antisymmetric representations of $S U(4)$. Since the Weyl fermions transform according to 4 and scalars according to 6 of the $R$-symmetry group, we have $a_{i j}^{\mathbf{4}}$ fermions $\Psi_{f_{i j}}^{i j}$ where $f_{i j}$ runs from 1 to $a_{i j}^{\mathbf{4}}$ and $a_{i j}^{\mathbf{6}}$ scalars $\Phi_{f_{i j}}^{i j}$ where $f_{i j}$ runs from 1 to $a_{i j}^{\mathbf{6}}$; all of these lie in the $\left(N_{i}, \bar{N}_{j}\right)$ representation of the group (in the scalar case we can use the reverse arrow contribution from $j$ to $i$ and thus think of these as complex fields).

Even though one can easily work out the general case, because we are motivated by the search for conformal theories we will be interested in a specific case where we choose the regular representation of $\Gamma$. In other words we start with an $U(n)$ gauge group, assume $n=N|\Gamma|$, and think of the fundamental representation of $U(n)$ as decomposing to the space $\mathbf{C}^{N} \otimes\{g\}$ with $g \in \Gamma$. We consider the action of $\Gamma$ to be on the second index as right multiplication. As is well known, this represention decomposes to a direct sum of all irreducible representions of $\Gamma$ with degeneracy factor $n_{i}=\operatorname{dim} \mathbf{r}_{i}$ for the $\mathbf{r}_{i}$ representation. In this case the gauge group we obtain is

$$
G=\otimes_{i} S U\left(N n_{i}\right)
$$

where the index $i$ runs over the irreducible representations of $\Gamma$. It is a straightforward exercise to show that the coupling constant $\tau_{i}$ of the i-th group (including the theta angle in the usual way) is given by

$$
\tau_{i}=\frac{n_{i} \tau}{|\Gamma|}
$$

where $\tau$ is the $\mathcal{N}=4$ coupling (or alternatively the type IIB coupling). Note in particular that

$$
\sum_{i} n_{i} \tau_{i}=\tau
$$

The matter content is naturally summarized by an associated "quiver" diagram. It consists of one node for each irreducible representation of $\Gamma$, and fermionic and bosonic arrows connecting the nodes according to how the 4- and 6-dimensional representations of $\Gamma$ (inherited from $S U(4)$ ) act on each irreducible representation. To each bosonic/fermionic 
arrow from node $i$ to node $j$ we associate a bifundamental $\left(N n_{i}, \overline{N n_{j}}\right)$ scalar/Weyl fermion (for $i=j$ this corresponds to an adjoint representation). There are Yukawa couplings for each triangle on the quiver consisting of two fermionic arrows and a bosonic arrow, and quartic scalar interactions for each square on the quiver consisting of four bosonic arrows. The coefficients of each interaction can be read off by projecting the original $\mathcal{N}=4$ lagrangian in terms of the fields we have kept. The Yukawa couplings are given by

$$
Y=\sum_{i j k} \gamma_{i j k}^{f_{i j}, f_{j k}, f_{k i}} \operatorname{Tr} \Psi_{f_{i j}}^{i j} \Phi_{f_{j k}}^{j k} \Psi_{f_{k i}}^{k i}
$$

and the quartic $\phi^{4}$ terms are given by

$$
V=\sum_{i j k l} \eta_{f_{i j}, f_{j k}, f_{k l}, f_{l i}}^{i j k l} \operatorname{Tr} \Phi_{f_{i j}}^{i j} \Phi_{f_{j k}}^{j k} \Phi_{f_{k l}}^{k l} \Phi_{f_{l i}}^{l i}
$$

where

$$
\gamma_{i j k}^{f_{i j}, f_{j k}, f_{k i}}=\Gamma_{\alpha \beta, m}\left(Y_{f_{i j}}\right)_{v_{i} \bar{v}_{j}}^{\alpha}\left(Y_{f_{j k}}\right)_{v_{j} \bar{v}_{k}}^{m}\left(Y_{f_{k i}}\right)_{v_{k} \bar{v}_{i}}^{\beta}
$$

and

$$
\eta_{f_{i j}, f_{j k}, f_{k l}, f_{l i}}^{i j k l}=\left(Y_{f_{i j}}\right)_{v_{i} \bar{v}_{j}}^{[m}\left(Y_{f_{j k}}\right)_{v_{j} \bar{v}_{k}}^{n]}\left(Y_{f_{k l}}\right)_{v_{k} \bar{v}_{l}}^{[m}\left(Y_{f_{l i}}\right)_{v_{l} \bar{v}_{i}}^{n]}
$$

Here summation over repeated indices is understood; $\left(Y_{f_{i j}}\right)_{v_{i} \bar{v}_{j}}^{\alpha},\left(Y_{f_{i j}}\right)_{v_{i} \bar{v}_{j}}^{m}$ are the $f_{i j}$ 'th Clebsch-Gordan coefficients corresponding to the projection of $4 \otimes \mathbf{r}_{i}$ and $6 \otimes \mathbf{r}_{i}$ onto $\mathbf{r}_{j}$; and $\Gamma_{\alpha \beta, m}$ is the invariant in $\mathbf{4} \otimes \mathbf{4} \otimes \mathbf{6}$.

Now we will ask whether the theory is conformal. As a first step towards proving this let us show that the one-loop beta-functions vanish. For the $i^{t h}$ factor $S U\left(N_{i}\right)$ in $G_{\text {proj }}$, there will generally be Weyl fermions transforming as:

$$
\oplus_{j} a_{i j}^{\mathbf{4}}\left(N_{i}, \bar{N}_{j}\right)
$$

and scalars transforming as

$$
\oplus_{j} a_{i j}^{\mathbf{6}}\left(N_{i}, \bar{N}_{j}\right)
$$

The one-loop beta-function for the gauge coupling $g_{i}$ is proportional to

$$
\beta_{i} \propto \frac{11}{3} N_{i}-\frac{1}{3} \sum_{j}\left(a_{i j}^{\mathbf{4}}+a_{i j}^{\overline{4}}\right) N_{j}-\frac{1}{2 \cdot 3} \sum_{j} a_{i j}^{\mathbf{6}} N_{j} .
$$


(note that this formula makes sense even if $j=i$ in the sum because in that case we get an adjoint representation and its second casimir is $N_{i}$ ). For $N_{i}=N n_{i}$ this expression vanishes, because

$$
\begin{gathered}
4 \cdot N_{i}=\sum_{j} a_{i j}^{4} N_{j}=\sum_{j} a_{i j}^{\overline{4}} N_{j} \\
6 \cdot N_{i}=\sum_{j} a_{i j}^{\mathbf{6}} N_{j}
\end{gathered}
$$

and

$$
\frac{11}{3}-\frac{2}{3} 4-\frac{1}{6} 6=0
$$

Of course this is far from proving that these theories correspond to conformal theories. However, considerations of [4] based on [3] strongly suggest they indeed are.

The considerations above apply to any discrete subgroup $\Gamma \subset S U(4)$. However, if $\Gamma \subset$ $S U(3)$ or $\Gamma \subset S U(2)$ we obtain $\mathcal{N}=1,2$ respectively. In these cases it is natural to consider a reduced quiver which still encodes the theory, taking into account the corresponding supersymmetry. For the $\mathcal{N}=1$ case, we consider the quiver with nodes given by irreducible representations of $\Gamma$, and consider $k$ arrows from the $i$-th node to $j$-th node if the $\mathbf{r}_{j}$ representation appears $k$ times in $\mathbf{3} \otimes \mathbf{r}_{i}$. These correspond to $k$ chiral multiplets $\Phi_{f_{i j}}^{i j}$ in the $\left(N_{i}, \bar{N}_{j}\right)$ representation. In this case we have a superpotential inherited from the $\mathcal{N}=4$ theory which is given by

$$
\mathcal{W}=\sum_{i, j, k} \sum_{f_{i j}, f_{j k}, f_{k i}} h_{i j k}^{f_{i j}, f_{j k}, f_{k i}} \operatorname{Tr}\left(\Phi_{f_{i j}}^{i j} \Phi_{f_{j k}}^{j k} \Phi_{f_{k i}}^{k i}\right)
$$

where

$$
h_{i j k}^{f_{i j}, f_{j k}, f_{k i}}=\epsilon_{\alpha \beta \gamma}\left(Y_{f_{i j}}\right)_{v_{i} \bar{v}_{j}}^{\alpha}\left(Y_{f_{j k}}\right)_{v_{j} \bar{v}_{k}}^{\beta}\left(Y_{f_{k i}}\right)_{v_{k} \bar{v}_{i}}^{\gamma}
$$

and $Y$ 's now correspond to Clebsch-Gordan coefficients for $\mathbf{3} \otimes \mathbf{r}_{i} \rightarrow \mathbf{r}_{j}$. Note that in this context the vanishing condition for the one loop beta function, $N_{f}=3 N_{c}$, follows from the fact that tensoring any representation $N_{c}$ with $\mathbf{3}$ gives a $3 N_{c}$ flavors (and that if we get adjoints they contribute like $N_{c}$ fundamentals).

It would be interesting to see whether this vanishing of beta function persists to higher loops. It is also important to check whether the anomalous scaling dimensions of the chiral fields are zero. In fact, the two are related [13] 14] [15]. We now show that the anomalous scaling dimensions at one loop vanish, which implies together with the vanishing of the gauge coupling beta functions at one loop that they continue to vanish at two loops. The 
matrix of anomalous scaling dimensions for chiral fields at one loop is given by (in our normalizations):

$$
\gamma_{a \bar{d}}^{(1)}=\sum_{b, c} h_{a b c} \bar{h}_{d b c}-2 \delta_{a \bar{d}}
$$

where the indices $a, b, c$ are what we called $f_{i j}, f_{j k}, f_{k i}, h_{a b c} \equiv h_{i j k}^{f_{i j}, f_{j k}, f_{k i}}$.

Denote by $W_{i j}$ the multiplicity spaces2: $\mathbf{3} \otimes \mathbf{r}_{i}=\oplus W_{i j} \otimes \mathbf{r}_{j}$, we also have $\overline{\mathbf{3}} \otimes \mathbf{r}_{i}=$ $\oplus W_{j i}^{*} \otimes \mathbf{r}_{j}$. The Yukawa coupling $h_{a b c}$ may be defined also as follows. consider the canonical element $\omega=\epsilon^{\alpha \beta \gamma} e_{\alpha} \otimes e_{\beta} \otimes e_{\gamma}$ of $\mathbf{3}^{3}$. The map $h: \mathbf{r}_{i} \rightarrow \mathbf{3}^{3}, h(v)=\omega \otimes v$ when expanded in the basis $Y_{a}$ of the spaces $W_{i j}$ etc. has the following form:

$$
h(v)=\sum_{a, b, c} h_{a b c} Y_{a} \otimes Y_{b} \otimes Y_{c} \otimes v
$$

where $Y_{a} \in W_{i j}, Y_{b} \in W_{j k}, Y_{c} \in W_{k i}$. Consider the following diagram:

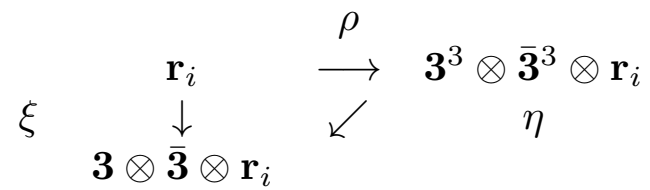

where $\rho(v)=\omega \otimes \bar{\omega} \otimes v$, for $v \in \mathbf{r}_{i}, \xi(v)=e_{\alpha} \otimes \bar{e}_{\alpha} \otimes v$ and $\eta(a \otimes b \otimes c \otimes \bar{d} \otimes \bar{e} \otimes \bar{f} \otimes v)=$ $\langle\bar{d}, c\rangle\langle\bar{e}, b\rangle a \otimes \bar{f} \otimes v$. The identity $\epsilon^{\alpha \beta \gamma} \epsilon^{\delta \beta \gamma}=2 \delta^{\alpha \delta}$ implies that

$$
\eta \circ \rho=2 \xi
$$

Now let us rewrite (2.11) in the basis $Y_{f_{i j}}$ of intertwiners. We shall use the indices $a$ for $f_{i j}, b$ for $f_{j k}$ and $c$ for $f_{k i}$. First of all, it is easy to represent the map $\rho$ as:

$$
\rho(v)=\sum_{a, b, c, d, e, f} h_{a b c} \bar{h}_{d e f} Y_{a} \otimes Y_{b} \otimes Y_{c} \otimes \bar{Y}_{f} \otimes \bar{Y}_{e} \otimes \bar{Y}_{d} \otimes v
$$

where $Y_{a} \in W_{i j}, Y_{b} \in W_{j k}, Y_{c} \in W_{k i}, \bar{Y}_{d} \in W_{m i}^{*}, \bar{Y}_{e} \in W_{n m}^{*}, \bar{Y}_{f}^{*} \in W_{i n}^{*}$ and we sum over $j, k, m, n$. Under the $\eta$ map the only vectors which are not mapped to zero are those for which $b=e, c=f$, hence $m=k, n=j$, hence

$$
\eta \circ \rho(v)=h_{a b c} \bar{h}_{d b c} Y_{a} \otimes \bar{Y}_{d} \otimes v
$$

On the other hand it is equal to $2 Y_{a} \otimes \bar{Y}_{a} \otimes v$, hence $\gamma_{a d}^{(1)}=0$.

2 We thank P. Etingof and D. Kazhdan for the explanation of this point 
If $\Gamma \in S U(2)$ we get an $\mathcal{N}=2$ theory. In this case the nodes of the quivers correspond to irreducible representations of $\Gamma$ and links correspond to the decomposition of representation upon tensoring with 2. This gives rise to the well known affine A-D-E Dynkin diagrams where the $n_{i}$ correspond to Dynkin indices associated with each node. In fact these $\mathcal{N}=2$ theories have been studied in [10] where it was shown that they are the only conformal $\mathcal{N}=2$ theories if the gauge group is a product of $S U$ 's and the matter is in the bifundamental representations. Note that the 2 in the conformality condition $N_{f}=2 N_{c}$ is the dimension of the fundamental representation of $S U(2)$. In fact, the power of orbifolding in this case suggests that perhaps even in the $\mathcal{N}=1$ and $\mathcal{N}=0$ cases considered above, in the subclass of gauge theories corresponding to product of $S U$ groups with matter in bifundamentals we have a full class of allowed conformal theories. It is quite interesting that discrete subgroups of $S U(3)$ [16] and $S O(6)$ [17] have already been classified. For example, for the $S U(3)$ case, in addition to the subgroups of $S U(2) \times U(1)$ one has: two infinite seria $\Delta\left(3 n^{2}\right)$ and $\Delta\left(6 n^{2}\right)$ (which are analogues of $A_{n}$ and $D_{n}$ 's in $S U(2)$ case - they are extensions of $\left.\mathbb{Z}_{n} \times \mathbb{Z}_{n}\right)$; and six exceptional cases $\Sigma(d), d=60,168,360 \varphi, 36 \varphi, 72 \varphi, 216 \varphi$, where $\varphi=1$ or 3 depending on whether the group belongs to $S U(3) / \mathbb{Z}_{3}$ or $S U(3)$ respectively. The number in braces is the order of the group.

It should be noted that a generic choice of $N_{i}$ s would not lead to a conformal theory. A conceptually useful example of a projection which does not lead to a superconformal theory is one for which the Chan-Paton factors transform in $n$ copies of a single irrep $\mathbf{r}_{\mathbf{1}}$ of $\Gamma \subset S U(2)$. It is easy to see that there will be no hypermultiplets after the projection and the theory will be a pure $S U(n) \mathcal{N}=2$ gauge theory, which is not superconformal.

\section{Relations to string theory}

If we realize these field theories via orbifolds of D3-brane theories, different choices of representations for the Chan-Paton factors will have definite physical meanings [5] [18] [7]. If we wish to describe $N$ D-branes away from the fixed point, the unprojected theory will be $U(N|\Gamma|)$ and the indices $I, J$ will lie in $N$ copies of the regular representation of $\Gamma$. This is in fact why we considered the action of $\Gamma$ in the previous section. Other representations will involve Chan-Paton factors living at the fixed point of the orbifold; they will correspond to D5- or D7- branes wrapped around shrunken 2- or 4-cycles [18][7]. Given the fact that these other physical projections make sense, it remains to explain why not all the orbifoldings give rise to conformal theories from the supergravity viewpoint [3]. 


\section{Moduli Space of Couplings in the Conformal Theory}

So far we have only considered the action induced from the $\mathcal{N}=4$ theory. This means that the conformal theory has at least one free coupling constant, which is that inherited from $\mathcal{N}=4$. It is natural to ask whether there are other deformations of this theory. In the case of $\mathcal{N}=2$, which corresponds to the ADE examples noted before, it is known that there is a moduli space of deformations, one coupling for each group. It was suggested in [ [ supergravity orbifolds. As noted in [4] there is a puzzle here as one would naively expect the blow-up modes to correspond to FI-terms. However we believe that in the present context 3-branes are secretly 5-branes wrapped around the extra vanishing two spheres, as has been suggested in [18][7] [19], in which case the couplings will be related to the expectation values of NS-NS $B_{i j}$ (related to coupling constant of gauge theory) and RR $B_{i j}$ (changing the theta angle) [5] fields.

As further confirmation of this picture, we now connect this picture to the known result that the moduli space of couplings for these theories is the moduli space of flat $A D E$ connections on the torus [10](for the $A$ case see also [20]). Let us first discuss more explicitly this moduli space. Let us consider flat $G$-connection $A_{z, \bar{z}}$ on a two-torus $E_{\tau}=\mathbb{C} /(\mathbb{Z}+\tau \mathbb{Z})$ with complex parameter $\tau$. Let $r$ be the rank of the group $G$ and let $H_{i}$ 's $i=1, \ldots, r$ be the generators of the Cartan subalgebra of $G$. It can be shown that by a complex gauge transformation the $(0,1)$ component $A_{\bar{z}}$ can be brought to a constant $\underline{\mathbf{t}}^{\mathbb{C}}$-valued form: $A_{\bar{z}}=\sum_{i} \frac{\tau_{i}}{\tau-\bar{\tau}} H_{i}$. Note that the $\tau_{i}$ are defined up to the action of the Weyl group of $G$. Moreover the gauge transformations of the form

$$
g(z, \bar{z})=\exp \left(\frac{2 \pi i}{\tau-\bar{\tau}}\left((z-\bar{z}) a_{i}+(z \bar{\tau}-\bar{z} \tau) b_{i}\right) H_{i}\right), \quad a_{i}, b_{i} \in \mathbb{Z}
$$

shift the value of $\tau_{i}$ by $a_{i}+b_{i} \tau$. We can introduce an extra $\tau_{0}$, obeying the property: $\sum_{i=0}^{r} n_{i} \tau_{i} \equiv \operatorname{0mod}(\mathbb{Z}+\tau \mathbb{Z})$ (where $n_{0}=1$ ). This presentation makes the action of $S L_{2}(\mathbb{Z})$ of the underlying torus manifest:

$$
\tau \rightarrow-1 / \tau, \quad \tau_{i} \rightarrow \tau_{i} / \tau
$$

Now we connect this to the moduli that we expect to see in the orbifold picture with the branes. By writing

$$
\tau_{i}=x_{i}+y_{i} \tau, \quad x_{i}, y_{i} \in \mathbb{R}, i=0, \ldots, r
$$


with the condition $\sum_{i} x_{i} \equiv \sum_{i} y_{i} \equiv 0 \bmod \mathbb{Z}$ we make the connection to the fluxes of the $B$-fields explicit: $\int_{\Sigma_{i}} B^{N S}=y_{i}, \int_{\Sigma_{i}} B^{R R}=x_{i}$. Here $\Sigma_{i}$ are the two-cycles of the corresponding ALE space, which are in one-to -one correspondence with simple roots of $G$. The action of $I I B S$-duality group on $B^{N S}, B^{R R}$ and string coupling $\tau$ translates to the action (4.2). We may also think of the $\tau_{i}$ 's as the points on the torus $E$ (actually, on the dual torus, which coincides with $E$ as complex manifold). The periodicity of the torus $x, y \sim x, y+1$ becomes the periodicity of the $B$-fields. The $\tau_{i}$ is the coupling for the $S U\left(N n_{i}\right)$ factor. The orbifold point corresponds to $x_{i}=0, y_{i}=\frac{n_{i}}{|\Gamma|}$. Note that the Weyl group action in the gauge theory setup is exactly mapped to the monodromy action in the context of ADE singularities. We thus see they are indeed identical.

It would be interesting to extend this to the case of $\mathcal{N}=1$ and $\mathcal{N}=0$, where again we may expect the 3 -brane to be secretly a combination of 5 -branes and 7 -branes. The moduli of the corresponding orbifold theory is expected to be related to the analogs of the various $B$ fields and the 4 -form gauge field expectation values. This should be interesting to develop and reconcile with the supergravity picture.

Another issue is the global identifications of the moduli space. This will correspond to non-trivial dualities in the field theory setup, which in the $\mathcal{N}=2$ is completely known. Since we do not know the full description of the moduli for the $\mathcal{N}=1$ and $\mathcal{N}=0$ cases we cannot specify the full duality group. However, there is already one complex modulus of the conformal theories which is inherited from the $\mathcal{N}=4$ coupling $\tau$ (which as noted before is related to the coupling of the gauge groups by $\left.\tau_{i}=\tau \frac{n_{i}}{|\Gamma|}\right)$. In this case the $S L(2, \mathbb{Z})$ invariance of Montonen-Olive will give rise to $S L(2, \mathbb{Z})$ symmetry in this subspace. In other words we thus expect to have a self-duality in this direction of coupling constant space. This is quite exciting, given that we are dealing with field theory S-dualities including the $\mathcal{N}=0$ case.

If our conjecture about the vanishing of beta functions in the $\mathcal{N}=0$ is true, this would indeed inovlve an amazing set of cancellations which is not a direct result of supersymmetry. It is natural to expect applications of this idea in the context of hierarchy problem. For example, in all of the models we have it is easy to see that we have equal numbers of bosons and fermions, even though we do not have supersymmetry. This is quite intriguing and may point to a new physical symmetry principle.

\section{Acknowledgements}


We would like to thank O. Bergman, P. Etingof, A. Johansen, D. Kazhdan, E. Silverstein and A. Strominger for valuable discussions. We are also grateful to Z. Kakushadze for pointing out the reference [9] as well as for discussions on the beta functions and to B. Zwiebach for bringing [1] to our attention.

This work was supported in part by NSF grant PHY-92-18167. In addition the research of N. N. was supported by Harvard Society of Fellows, partially by RFFI under grant 96-02-18046 and partially by grant 96-15-96455 for scientific schools. 


\section{References}

[1] S. Hamidi, J.Patera, J. Schwarz, "Chiral Two-Loop Finite Supersymmetric Theories", Phys. Lett. 141B (1984) 349

[2] S. Gubser and I. Klebanov, "Absorption by Branes and Schwinger Terms in the World Volume Theory," Phys. Lett. B413 (1997) 41.

[3] J. Maldacena, "The Large-N Limit of Superconformal Field Theories and Supergravity", hep-th/9712200

[4] S. Kachru and E. Silverstein, "4d Conformal Field Theories and Strings on Orbifolds", hep-th/9802183

[5] M.R. Douglas and G. Moore, "D-branes, Quivers and ALE Instantons", hepth/9603167

[6] C.V. Johnson and R.C. Myers, "Aspects of Type IIB Theory on ALE Spaces", Phys. Rev. D55 (1997) 6382, hep-th/9610140

[7] M.R. Douglas, "Enhanced Gauge Symmetry in M(atrix) theory", JHEP 007(1997) 004; hep-th/9612126

[8] M.R. Douglas, B.R. Greene and D.R. Morrison, "Orbifold Resolution by D-branes", Nucl. Phys. B505 (1997) 84; hep-th/9704151

[9] L.E. Ibáñez, "A Chiral D=4, N=1 String Vacuum with a Finite Low Energy Effective Field Theory," hep-th/9802103

[10] S. Katz, P. Mayr and C. Vafa, "Mirror Symmetry and Exact Solution of 4D $\mathcal{N}=2$ Gauge Theories - I", hep-th/9706110

[11] N. Seiberg, "Notes on Theories with 16 Supercharges", hep-th/9705117

[12] E.G. Gimon and J. Polchinski, "Consistency Conditions for Orientifolds and D Manifolds", Phys. Rev. D54 (1996) 1667, hep-th/9601038

[13] D. R. T. Jones and L. Mezincescu, "The Beta Function in Supersymmetric Yang-Mills Theory", Phys. Lett. B137 (1984) 242;

P. West, "The Yukawa Beta-Function in $\mathcal{N}=1$ Rigid Supersymmetric Theories", Phys. Lett. 137B (1984) 371;

A. Parkes and P. West, "Finiteness in Rigid Supersymmetric Theories" Phys. Lett. 138B (1984) 99;

D. R. T. Jones and L. Mezincescu, "The Chiral Anomaly and a Class of Two-Loop Finite Supersymmetric Gauge Theories", Phys. Lett. 138B (1984) 293

[14] M. Shifman and A. Vainshtein, "Solution of the Anomaly Puzzle in Susy Gauge Theories and the Wilson Operator Expansion", Nucl. Phys. B277 (1986) 456 ;

"On Holomorphic Dependence and Infrared Effects in Supersymmetric Gauge Theories", Nucl. Phys. B359 (1991) 571

[15] R. G. Leigh, M.J. Strassler, "Exactly Marginal Operators and Duality in Four Dimensional N=1 Supersymmetric Gauge Theory", Nucl. Phys. B447 (1995) 95 
[16] W.M. Fairbanks, T. Fulton and W.H. Klink, "Finite and Disconnected Subgroups of $S U(3)$ and their Application to the Elementary Particle Spectrum", J. Math Phys. 5 (1964) 1038

[17] W. Plesken and M. Pohst, Math. Comp. 31 (1977) 552

[18] J. Polchinski, "Tensors from K3 Orientifolds", Phys. Rev. D55 (1997) 6423; hepth/9606165

[19] D.-E. Diaconescu, M.R. Douglas and Jaume Gomis, "Fractional Branes and Wrapped Branes", hep-th/9712230

[20] E. Witten, "Solutions of Four-dimensional Field Theories via M-theory", Nucl. Phys. B500 (1997) 3; hep-th/9703166 